\title{
Aspectos reproductivos y pesqueros de Prochilodus magdalenae Steindachner, 1879 (Characiformes: Prochilodontidae) en la ciénaga de Marriaga, río Atrato, Colombia
}

\author{
(1) Margarita María Roa-Cubillos*, Francisco Antonio Villa-Navarro
}

Grupo de Investigación en Zoología, Departamento de Biología, Facultad de Ciencias, Universidad del Tolima, Ibagué, Colombia

\begin{abstract}
Resumen
Prochilodus magdalenae ha sido históricamente una de las fuentes de alimento de mayor importancia para la población a nivel nacional. Esta especie endémica de Colombia ha proporcionado más del $50 \%$ del volumen de capturas en las principales cuencas trasandinas del país. El aislamiento de la población de $P$. magdalenae en la cuenca del Atrato hace necesario que se establezcan medidas de conservación ajustadas a sus características biológicas y reproductivas. En el presente estudio se registró información biométrica de las capturas de $P$. magdalenae provenientes de la pesca artesanal en la ciénaga de Marriaga (cuenca baja del río Atrato) desde abril de 2014 a marzo de 2015. Se registraron 574 individuos de los cuales 427 eran hembras y 147, machos. La distribución de las tallas para las hembras estuvo entre 200 y $470 \mathrm{~mm}$ de longitud estándar (LE) y para los machos entre 210 y $415 \mathrm{~mm}$. El análisis de la longitud y el peso evidenció un crecimiento isométrico $(b=2,9577)$ y una gran correlación entre las dos variables $\left(\mathrm{r}^{2}=0,8119\right)$ para las hembras, en tanto que para los machos el crecimiento fue alométrico negativo $(b=1,8793)$ con una correlación baja $\left(\mathrm{r}^{2}=0,5628\right)$. La talla media de madurez $(\mathrm{TMM})$ para la población total fue de $365 \mathrm{~mm}$ (LE). La época de mayor actividad reproductiva se estimó entre octubre y mayo. La distribución de las tallas y la talla media de madurez (TMM), así como las épocas de desove, difirieron de las registradas en las zonas baja y media del Atrato, así como en las otras cuencas trasandinas. (C) 2019. Acad. Colomb. Cienc. Ex. Fis. Nat.
\end{abstract}

Palabras clave: Prochilodus magdalenae; Río Atrato; Aspectos reproductivos; Aspectos pesqueros.

Reproductive aspects and fishery of Prochilodus magdalenae Steindachner, 1879 (Characiformes: Prochilodontidae) in the Marriaga swamp, Atrato River system, Colombia

\begin{abstract}
Prochilodus magdalenae has historically been one of the most important food sources for the human population nationwide. This Colombian endemic species represents more than $50 \%$ of all catches in the main river basins of the country. The isolation of the population of $P$. magdalenae in the Atrato basin demands the adoption of conservation measures adapted to their biological and reproductive characteristics. During our study we recorded the biometric information of P. magdalenae catches obtained via artisanal fishing in the Marriaga swamp (Atrato River lower basin) from April 2014 to March 2015. From a total of 574 recorded individuals, 427 were females and 147 males. The female size ranged between 200 and $415 \mathrm{~mm}$ (standard length, SL), and the male size ranged between 210 and $415 \mathrm{~mm}$ (SL). In females, the length-weight analysis indicated an isometric growth $(b=2.95)$ and a high correlation between the two variables $\left(\mathrm{r}^{2}=0.81\right)$. In males, it indicated a negative allometric growth $(b=1.87)$ and a low correlation $\left(\mathrm{r}^{2}=0,56\right)$. The average size at maturity for the whole analyzed population was $365 \mathrm{~mm}$ (SL). The highest period of reproductive activity was estimated between October and March. The size ranges, the average size at maturity, and the spawning seasons of the individuals registered in the Marriaga swamp differed from those registered in the middle and lower area of the Atrato river basin, and other basins where the $P$. magdalenae has been studied. (C) 2019. Acad. Colomb. Cienc. Ex. Fis. Nat.
\end{abstract}

Key words: Prochilodus magdalenae; Atrato River; Reproductive aspects; Fishing aspects.

\section{Introducción}

El bocachico (Prochilodus magdalenae Steindachner, 1879) es la especie pesquera continental de mayor importancia comercial en Colombia; durante décadas ha sido parte de la seguridad alimentaria de la población colombiana y fuente de empleo para los pescadores pertenecientes a las cuencas de los ríos Magdalena, Sinú y Atrato (Galvis \&
Mojica, 2007; Valderrama, et al., 2011). Dado que es una de las especies más investigadas en la cuenca del río Magdalena (Valderrama, et al., 2011), ha sido un referente para

\footnotetext{
*Correspondencia:

Margarita María Roa-Cubillos; margararoa@gmail.com

Recibido: 7 de septiembre de 2018

Aceptado: 12 de febrero de 2019

Editor: Arturo Acero
} 
establecer las medidas de manejo que se aplican actualmente para sus poblaciones en las diferentes cuencas del país (Mojica, et al., 2012).

Prochilodus magdalenae se encuentra en la categoría de vulnerable (VU A2c,d) debido a la fuerte presión pesquera a que se ha visto sometido en los últimos 40 años (Mojica, et al., 2012). En la cuenca del río Magdalena su participación pesquera en 1970 fue de 63,64 \% (17.945,8 ton/ año) y para 2009 había descendido a 37,8 \% (Gutiérrez, et al., 2011). Esta presión pesquera también se evidencia con el seguimiento de su talla de captura: en 1973 la talla media de captura (TMC) se estableció en $38 \mathrm{~cm}$ de longitud estándar (LE), en 1987 se redujo a $27 \mathrm{~cm}$ (Mojica, et al., 2012) y, actualmente ha descendido a $24,78 \mathrm{~cm}$ (Alonso, et al., 2014).

En la cuenca del Sinú las capturas de $P$. magdalenae entre 1997 y 2002 representaron el $34 \%$ de la captura total (promedio 645 toneladas/año), pero a finales de la primera década de este siglo sus valores anuales disminuyeron a menos de $<400$ toneladas/año (Valderrama, et al., 2011). La TMC entre 2001 y 2002 se estableció en $23,35 \mathrm{~cm}$ de LE (Valderrama, et al., 2002) y se redujo a $20,86 \mathrm{~cm}$ de LE entre el año 2012 y 2013 (Alonso, et al., 2014). Por otra parte, la talla media de madurez (TMM) descendió de 25,3 cm de LE en el 2003 (Valderrama \& Solano, 2004) a 22,48 cm en tan solo una década (Alonso, et al., 2014).

Los estudios publicados sobre la cuenca del río Atrato son pocos y relativamente recientes. En la cuenca media, $P$. magdalenae ha representado el $47 \%$ de las capturas (Rivas, et al., 2002), y el 52,08 \% en la cuenca baja durante la década de los años 80 del siglo pasado (Mora, 1990), en tanto que más recientemente se ha estimado que constituye cerca del $80 \%$ de los desembarcos registrados en Quibdó (Gutiérrez, et al., 2011). El volumen de los desembarcos se ha registrado con regularidad en la cuenca media, donde durante el período de 1997 a 2010 fluctuó entre 400 y 5.000 toneladas/año (Gutiérrez, et al., 2011); sin embargo, al final de este período dicho volumen disminuyó en un $50 \%$ con respecto a los valores iniciales (Valderrama, et al., 2011). Por otra parte, la TMC se ha reducido durante los últimos años, pasando de 24,9 cm de LE (Mora, 1990) a 23,5 cm (Valderrama, et al., 2011).

El desconocimiento de la biología de una cohorte afecta su manejo adecuado, pues se establecen tallas mínimas de captura o épocas de veda que pueden no corresponder a las características propias de la población que requiere un manejo sustentable. En este sentido, el objetivo del presente estudio fue determinar las características reproductivas y poblacionales de los individuos de $P$. magdalenae de la ciénaga de Marriaga, cuenca baja del río Atrato, Colombia.

\section{Materiales y métodos}

La cuenca del río Atrato abarca $38.000 \mathrm{~km}^{2}$, tiene una longitud de $750 \mathrm{~km}$ y su llanura aluvial cubre 130.000 ha. La cuenca presenta un régimen hidrológico unimodal, con una época de lluvias intensas entre mayo y noviembre y de menor intensidad entre diciembre y abril (Jaramillo-Villa \& Jiménez-Segura, 2008).

La ciénaga de Marriaga se localiza en las coordenadas $08^{\circ} 06^{\prime} 38,0^{\prime \prime} \mathrm{N}$ y $76^{\circ} 57^{\prime} 50,4^{\prime}$ ' O, cerca de la desembocadura del río Atrato en el Golfo de Urabá (Figura 1). Forma un complejo lagunar con la ciénaga Limón, protegida de la extracción de recursos pesqueros, y hace parte de la zona de amortiguación del Parque Nacional Natural de Los Katios (PNN Katios, 2006).

La ciénaga de Marriaga está ubicada en jurisdicción del municipio de Unguía, departamento de Chocó, entre los 0 y los $12 \mathrm{~m} \mathrm{~s}$. n. m., próxima a la desembocadura del río Atrato en el mar Caribe, por lo que tiene influencia de las aguas marinas y está categorizada como laguna costera (INVEMAR, 2002). Además de presentar macrófitas flotantes en el espejo de agua, con dominio de Eichornia crassipes, las asociaciones vegetales características de esta zona son principalmente arracachales (Montrichardia arborescens), panganales (Raphia taedigera), manglares de mangle rojo (Rhizophora mangle) y negro (Avicennia germinans) y cativales (Prioria copaifera) (Codechocó, 2013).

Los datos pluviométricos durante el periodo de estudio (2014-2015), así como el promedio mensual 'multianual' de la década anterior al estudio (2005-2014), se obtuvieron a partir de los registros de la estación meteorológica Unguía (11130010) colocada en la cuenca baja de río Atrato por el Instituto de Hidrología, Meteorología y Estudios Ambientales de Colombia (IDEAM) (Figura 2).

Los ejemplares de $P$. magdalenae fueron recolectados por pescadores artesanales entre abril de 2014 y marzo de 2015 utilizando la técnica de trasmallo con redes de multifilamento de ojo de malla variable entre 7,6 y $12,7 \mathrm{~cm}$, una altura de 2,5 a $7 \mathrm{~m}$ y una longitud de 100 a $1.000 \mathrm{~m}$. Se determinó la longitud total (LT: $\mathrm{cm})$ de cada individuo muestreado, su longitud estándar (LE: cm), el peso total (W: g) y su estadio de madurez sexual (EMS), en tanto que en una submuestra se registró el peso gonadal (g). Estas muestras se fijaron en formol al $10 \%$ para análisis posteriores.

Análisis estadístico. La distribución de las tallas de los ejemplares se obtuvo empleado los valores de la LE agrupada en intervalos de $2 \mathrm{~cm}$, y se graficó con las marcas de clase para cada intervalo. El análisis de la relación de longitud y peso se hizo tanto para la población total como discriminando en hembras y machos, y los datos se agruparon de forma bimensual para así utilizar esta relación matemática como una estimación indirecta de la tasa de crecimiento (Gonzáles, et al., 1996). Dicha relación se estableció empleando la ecuación:

$$
W=a L^{b}
$$

donde $W$ es el peso total (g), y $L$ la longitud estándar (LE $\mathrm{cm})$, y $a$ y $b$ son constantes (Froese, 2006).

Se determinó la proporción sexual total y bimensual con la información de campo de los ejemplares recolectados y con esos datos se probó la hipótesis de una proporción 


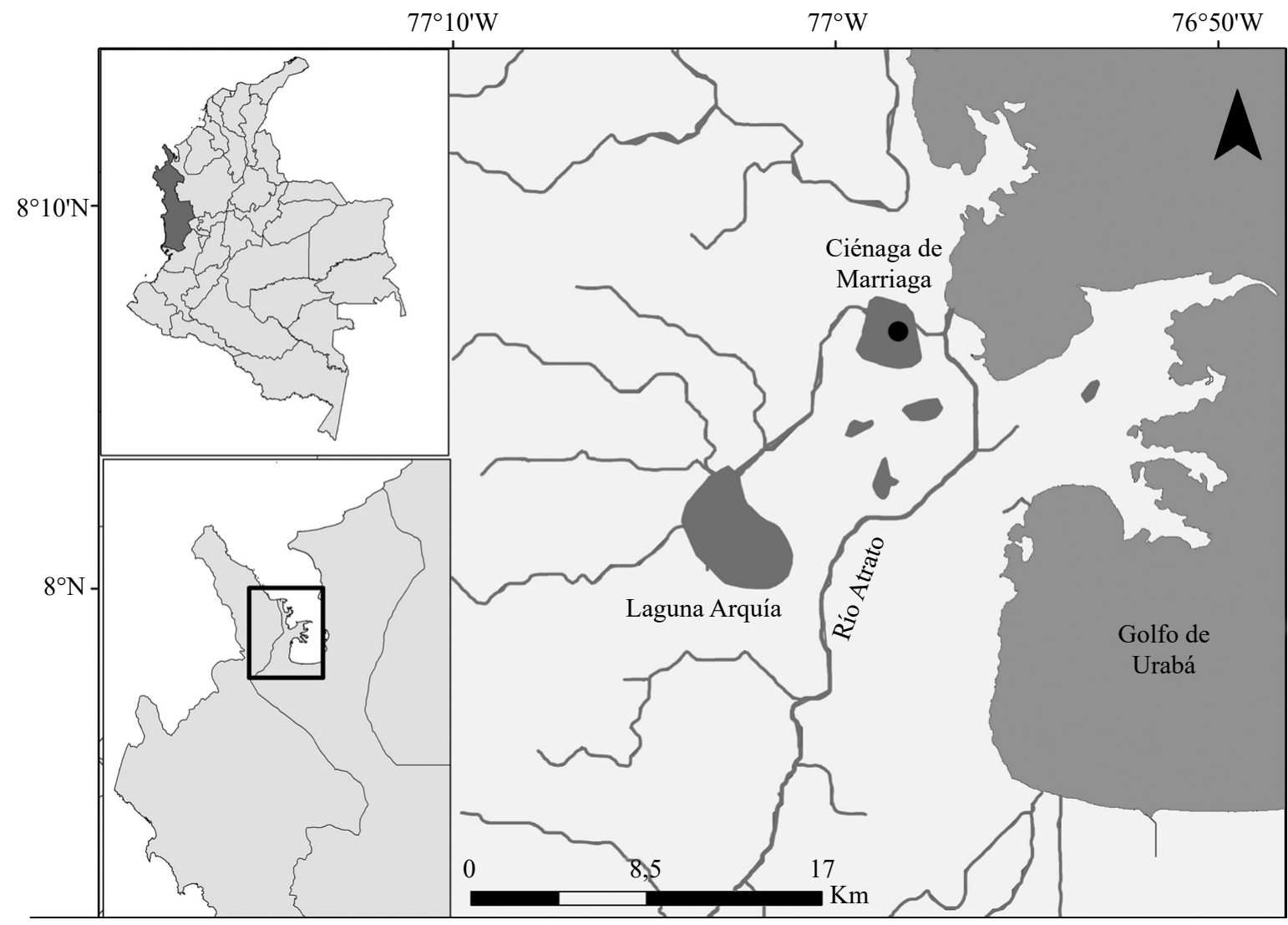

Figura 1. Localización geográfica del área de estudio (ciénaga de Marriaga) entre los departamentos de Chocó y Antioquia, y próxima a la desembocadura del río Atrato en el mar Caribe, Colombia

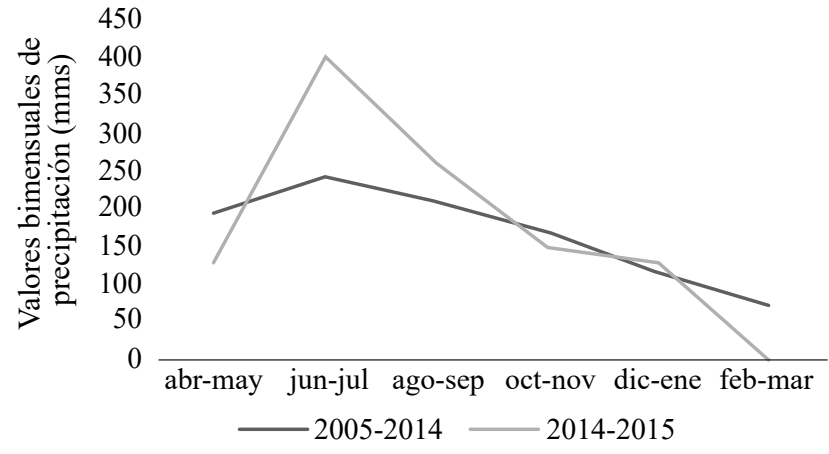

Figura 2. Valores bimensuales 'multianuales' de la década de 2005 a 2014 y del año de estudio (2014-2015) registrados en la estación meteorológica Unguía (11130010) del Instituto de Hidrología, Meteorología y Estudios Ambientales de Colombia (IDEAM)

sexual de 1:1 mediante la prueba de ji al cuadrado. Solo se emplearon las hembras como indicador de los periodos de reproducción para analizar la dinámica reproductiva (Wootton, 1995).

El análisis macroscópico de las gónadas se hizo según la propuesta de Vazzoler (1996): (I) inmaduros, (II) madurando, (III) maduros y (IV) desovados. Asimismo, se analizó la variación mensual del índice gonadosomático
(IGS), en el cual el peso de las gónadas se expresa como un porcentaje del peso corporal. Se utilizó el análisis de varianza (ANOVA) de Kruskal-Wallis $(\mathrm{p}<0,05)$ para probar la hipótesis de que el IGS no varía durante un ciclo hidrológico y determinar dónde se encontraba la diferencia en caso de que esta existiera. Para determinar la posible relación entre la pluviosidad y el IGS, se empleó la prueba de correlación de Spearman $(\mathrm{p}<0,05)$.

La talla media de madurez (TMM) se estableció empleando la LE a la cual correspondía el $50 \%$ de la frecuencia acumulada de individuos maduros (EMS III) mediante la siguiente ecuación:

$$
S(L)^{\prime}=\frac{1}{\left[1+e^{(s 1-s 2 L)}\right]}
$$

donde $L=$ longitud, $S(L)^{\prime}=$ frecuencia relativa acumulada corregida y $S 1$ y $S 2$ representan constantes iguales a las constantes $a$ y $b$ obtenidas de la regresión lineal entre $L n[1 / S(L)-1)$ y $L$ (Sparre \& Venema, 1997).

Para analizar el crecimiento de la población se utilizó la ecuación de crecimiento de von Bertalanffy (1938), donde $L t$ es igual a la longitud a la edad t, $L_{\infty}$ es la longitud asintótica, $t_{o}$ es la edad hipotética del pez en la que su longitud equivale a cero y $k$ es el parámetro de crecimiento; para ello se utilizó la información proveniente de la frecuencia de tallas con el empleo del programa FISAT II. 


$$
L t=L_{\infty}\left[1-e^{-K\left(t-t_{0}\right)}\right]
$$

En los casos en los que se presentaba crecimiento oscilatorio se utilizó la ecuación propuesta por Pauly (1979) donde $C$ es la amplitud de la oscilación:

$$
L t=L_{\infty}\left[1-e^{-K\left(t-t_{0}\right)+c\left(\frac{K}{2 \pi}\right) \operatorname{sen} 2 \pi(t-t s)}\right]
$$

La explotación pesquera se estimó mediante el cálculo de la mortalidad total $(Z)$ con el método de curva de captura linealizada en su versión modificada para convertir las tallas en edades, para lo cual se utilizó el programa FISAT II (Gayanilo \& Pauly, 1997):

$$
t(L)=t_{0}-\frac{1}{K} * \ln \left(1-\frac{L}{L_{\infty}}\right),
$$

donde $N$ es el número de peces en cada clase de talla, $\Delta t$ es el tiempo necesario para que el pez pase de una clase de talla a la siguiente, y $t$ es la edad correspondiente a la marca de clase $i$.

$$
\ln \left(\frac{N_{i}}{\Delta \mathrm{t}_{i}}\right)=a+b * t_{i}
$$

La información se analizó de manera bimensual con el fin de agrupar un número representativo de datos. Las pruebas estadísticas se hicieron con el paquete estadístico InfoStat, versión 2013 (Di Rienzo, et al., 2013) y un nivel de significación de $\alpha=0,05$.

\section{Resultados}

Se registró un total de 580 ejemplares de $P$. magdalenae, de los cuales 426 fueron hembras y 144 machos. Las tallas estuvieron entre 20,0 y $47,0 \mathrm{~cm}$ de $\mathrm{LE}(\dot{\mathrm{x}}=32,1 \mathrm{~cm}$; $\mathrm{s}=5,9$ ), donde el intervalo de longitud con mayor número de individuos estuvo comprendido entre 25,0 y $27,0 \mathrm{~cm}$ de LE (n=112) (Figura 1S, https:/www.raccefyn.co/index. php/raccefyn/article/downloadSuppFile/729/2966). En las hembras $(n=426)$ los valores estuvieron entre 20,0 y $47,0 \mathrm{~cm}$ de LE $(\dot{x}=33,1 \mathrm{~cm} ; \mathrm{s}=5,9)$ y en los machos $(\mathrm{n}=144)$ entre $21,0$ y $41,5 \mathrm{~cm}(\dot{\mathrm{x}}=29,5 \mathrm{~cm} ; \mathrm{s}=4,6)$ (Figura 3$)$.

La relación entre longitud y peso de la población total se describió mediante el modelo exponencial con la siguiente ecuación: $\mathrm{W}_{\mathrm{t}}=0,035 \mathrm{~L}^{2,8536} \mathrm{y} \mathrm{r}^{2}=0,779$, y con límites de confianza (LC $95 \%$ ) para $b$ entre 2,63 y 3,12, lo que indicaba un crecimiento isométrico. La ecuación utilizada en el caso de las hembras fue $\mathrm{W}=0,024 \mathrm{~L}^{2,9577}$ (LC $95 \%$ ), para una $b$ entre 2,83 y 3,09 y una $a$ entre 0,014 y 0,038 , lo que indicaba un crecimiento isométrico. En los machos la ecuación fue $\mathrm{W}=0,801 \mathrm{~L}^{1,8793}$ (LC $95 \%$ ), para una $b$ entre 1,61 y 2,15 y una $a$ entre 0,319 y 2,013 , lo cual correspondía a valores de crecimiento alométrico negativo (Figura 2S, https:/www.raccefyn.co/index.php/raccefyn/ article/downloadSuppFile/729/2967) (Tabla 1).
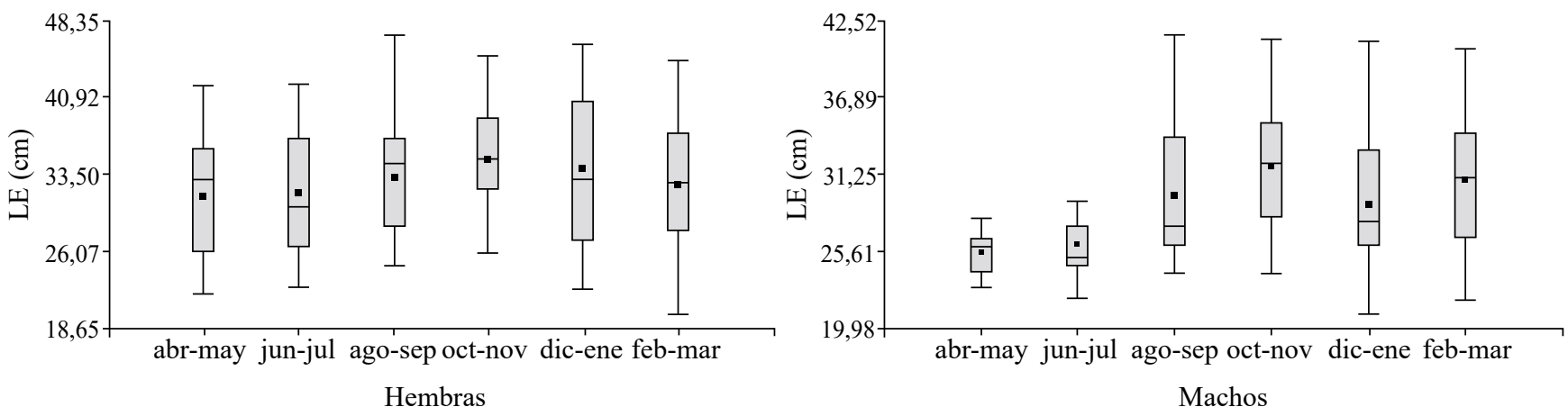

Figura 3. Distribución de tallas de hembras y machos de $P$. magdalenae capturados en la ciénaga de Marriaga, cuenca baja del río

\begin{tabular}{|c|c|c|c|c|c|c|c|c|}
\hline \multirow[t]{2}{*}{ Bimestre } & \multicolumn{4}{|c|}{ Hembras } & \multicolumn{4}{|c|}{ Machos } \\
\hline & $\mathbf{n}$ & $\boldsymbol{B}$ & LI-LS (95\%) & $\mathbf{r}^{2}$ & $\mathbf{N}$ & B & LI-LS (95\%) & $\mathbf{r}^{2}$ \\
\hline Abril - mayo & 81 & 2,712 & $2,490-2,960$ & 0,8529 & 9 & 1,479 & $-1,980-4,930$ & 0,4519 \\
\hline Junio - julio & 49 & 3,005 & $2,620-3,390$ & 0,8405 & 15 & 0,806 & $-0,360-1,970$ & 0,1463 \\
\hline Agosto - septiembre & 59 & 2,914 & $2,600-3,230$ & 0,8551 & 31 & 1,948 & $1,470-2,430$ & 0,7034 \\
\hline Octubre - noviembre & 83 & 3,432 & $3,050-3,810$ & 0,8012 & 31 & 1,847 & $1,060-2,640$ & 0,4395 \\
\hline Diciembre - enero & 97 & 3,092 & $2,800-3,380$ & 0,8265 & 40 & 1,977 & $1,510-2,440$ & 0,6588 \\
\hline Febrero - marzo & 57 & 2,817 & $2,440-3,190$ & 0,8057 & 18 & 2,143 & $1,660-2,630$ & 0,8471 \\
\hline
\end{tabular}
Atrato, Colombia

Tabla 1. Valores bimensuales resultantes de la relación entre longitud y peso de hembras y machos de P. magdalenae capturados en la ciénaga de Marriaga, Colombia.

$b=$ pendiente, $\mathrm{r}^{2}=$ coeficiente de determinación, LI-LS $(95 \%)=$ límite inferior-límite superior 
Los machos representaron $25,5 \%(\mathrm{n}=144)$ y las hembras $74,5 \%(\mathrm{n}=426)$ de la captura total, es decir, una proporción de $1: 3\left(\chi^{2}=13,64 ; p=0,0181\right)$. Esta proporción varió durante el periodo de estudio, con una proporción máxima entre machos $(\mathrm{M})$ y hembras $(\mathrm{H})$ de 1:7 entre abril y mayo y una mínima de 1:1,9 entre agosto y septiembre (Figura 4). La proporción de $\mathrm{M}: \mathrm{H}$ durante abril y mayo presentó diferencias significativas con respecto a los otros meses $(p<0,05)$.

Se registraron hembras en EMS III entre octubre y mayo, siendo el periodo de febrero a marzo el de mayor captura de ejemplares maduros; además, se registraron ejemplares desovados y en reposo (EMS IV) durante todo el año. La presencia de machos maduros (EMS III) se registró entre agosto y marzo, y disminuyó durante los últimos meses de la etapa reproductiva. Se capturaron individuos en reposo (EMS IV) solo durante febrero y marzo, en tanto que entre abril y julio no se capturó ningún individuo adulto (Figura 5).

El IGS para las hembras presentó un mayor valor entre febrero y marzo $(\dot{\mathrm{x}}=1,07 ; \mathrm{s}=1,54)$ y menor entre agosto y septiembre $(\dot{x}=0,25 ; s=0,25)$; según el ANOVA de Kruskal-Wallis hubo diferencias significativas entre

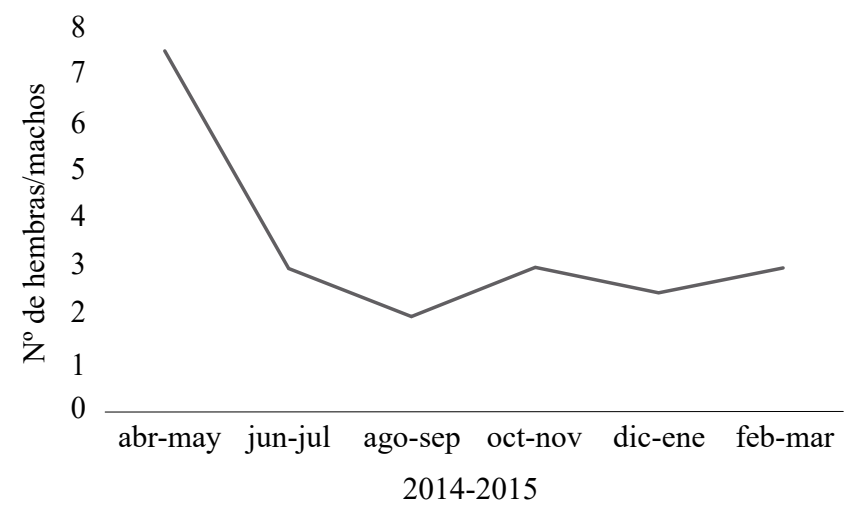

Figura 4. Proporción de hembras y machos de $P$. magdalenae en los ejemplares capturados durante el periodo de estudio en la ciénaga de Marriaga, cuenca baja del río Atrato, Colombia agosto y septiembre y el resto del año $(\mathrm{p}=0,045)$ (Figura 3S, https://www.raccefyn.co/index.php/raccefyn/article/ downloadSuppFile/729/2968). Asimismo, el análisis de correlación de Spearman mostró una relación significativa entre los valores de pluviosidad y la variación de la actividad reproductiva (IGS) bimensual $(\mathrm{p}<0,05)$. El análisis para establecer la talla media de madurez (TMM) demostró que el $50 \%$ de los individuos de la población alcanzaba la madurez al llegar a los 36,5 cm de LE. Las hembras alcanzaron una TMM al llegar a los $37,0 \mathrm{~cm}$ de LE, y los machos con $35,0 \mathrm{~cm}$ de LE (Figura 6).

La estimación de la tasa de mortalidad evidenció una mortalidad natural de 0,97 , un coeficiente de mortalidad por pesca (F) de 0,46 y una tasa de explotación $€$ de 0,32 , en tanto que su tasa instantánea de mortalidad (Z) fue de 1,43 (Figura 4S, https://www.raccefyn.co/index.php/raccefyn/ article/downloadSuppFile/729/2969).

Con una longitud asintótica $\left(\mathrm{L}_{\mathrm{oo}}\right)$ de 53,0, una amplitud de la oscilación (C) de 0,1 y un punto de invierno (WP) de 0,95 , la población presentó una tasa de crecimiento $(\mathrm{K})$ de $0,520 \mathrm{año}^{-1}$. La ecuación de crecimiento hallada fue:

$$
L_{t}=53,0\left(1-\mathrm{e}^{-0,520(t-t)-0,1 / 2 \pi \operatorname{sen} 0,9}\right) \text {. }
$$

El gráfico de tallas y la curva de crecimiento (Figura 5S, https://www.raccefyn.co/index.php/raccefyn/article/ downloadSuppFile/729/2970) evidenciaron que la población presentaba un crecimiento oscilatorio, con un tiempo de vida estimado de cuatro años, y que el reclutamiento podría efectuarse durante junio.

\section{Discusión}

Las tallas de los ejemplares de $P$. magdalenae capturados en la ciénaga de Marriaga difirieron de las registradas en la misma cuenca y otras cuencas del país, lo que puede atribuirse a diferentes presiones de pesca o a dinámicas poblacionales distintas. Se considera que la disminución del tamaño de los peces, así como su maduración acelerada, es una respuesta a la presión pesquera en sus poblaciones (Sparre \& Venema,
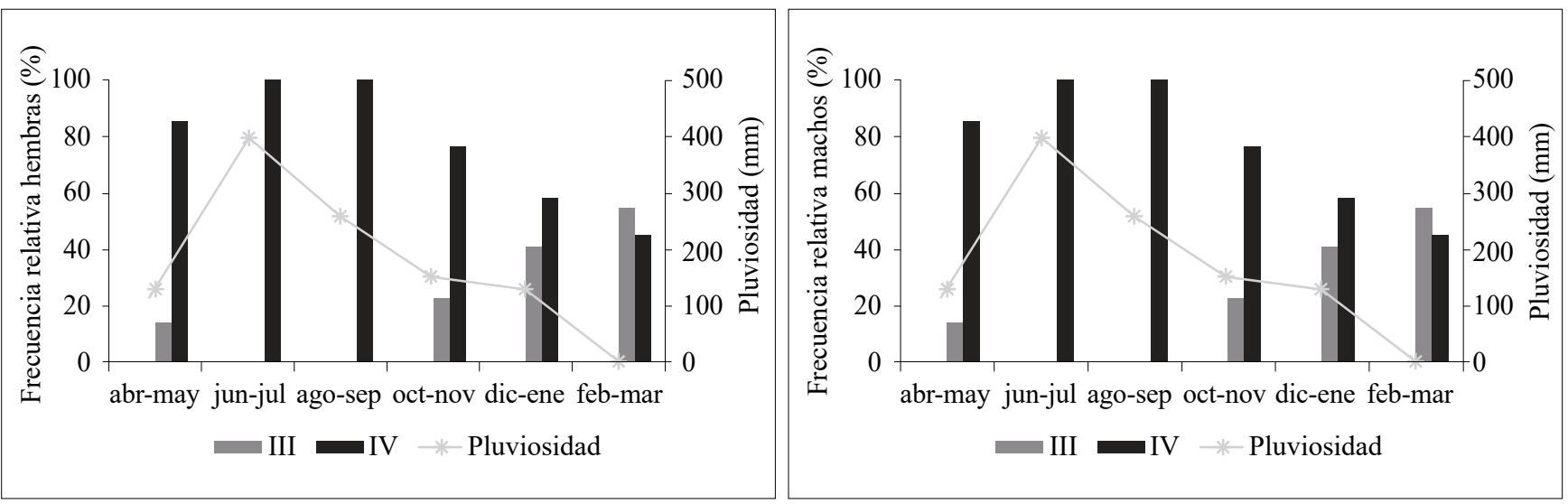

Figura 5. Frecuencia relativa de los estadios de madurez sexual III y IV de hembras y machos de $P$. magdalenae capturados durante el periodo de estudio en la ciénaga de Marriaga, cuenca baja del río Atrato, Colombia 

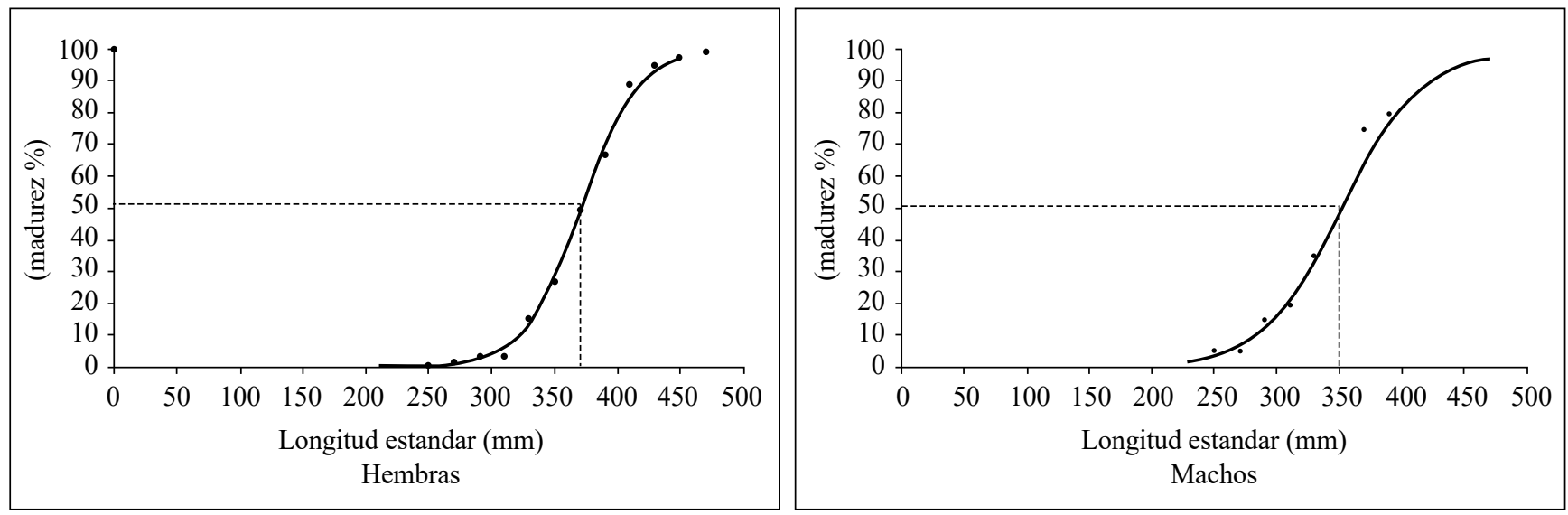

Figura 6. Talla media de madurez (TMM) (línea punteada) de hembras y machos de P. magdalenae de la ciénaga de Marriaga, cuenca baja del río Atrato, Colombia

1997; Welcomme, 1999; Allan, et al., 2005), lo que sugiere que la mayor TMM registrada en la ciénaga de Marriaga obedecería a una menor presión pesquera ejercida sobre la especie en este sector del río Atrato.

Prochilodus magdalenae es una especie migratoria, cuya reproducción es anual y se sincroniza con el régimen hidrológico de la cuenca (Maldonado-Ocampo, et al., 2012). Durante el periodo de estudio la época reproductiva se registró en los meses de menor pluviosidad (octubre a mayo), lo cual fue evidente por la presencia de individuos maduros (EMS III), así como por el alto valor del IGS, el cual se relaciona con el desarrollo y el crecimiento del ovario y es un indicador preciso de las épocas de reproducción (Vazzoler, 1996). Los datos difirieron de los registrados por otros autores para la misma cuenca, ya que en sus estudios se estableció que entre noviembre y diciembre se iniciaba la maduración gonadal con desoves entre abril y mayo, aunque estos resultados corresponden a estudios parciales (Jaramillo-Villa \& Jiménez-Segura, 2008) y en la zona media del Atrato (Cala \& Román-Valencia, 1999).

La relación entre longitud y peso reflejó diferentes tipos de crecimiento en machos y hembras, lo cual podría atribuirse a diferencias de edad entre los ejemplares de cada sexo capturados. Durante el estudio se registró un mayor número de machos en estadio juvenil que hembras en este mismo estadio. Por otra parte, los valores menores de $b$ después del pico reproductivo podrían reflejar la inversión energética que implica la producción de gónadas y la época de apareamiento (Froese, 2006), así como el desplazamiento de individuos adultos después de este pico, principalmente de machos, cuyas tallas y valores de $b$ reflejan la presencia exclusiva de individuos juveniles en la ciénaga.

La ausencia de machos de tallas grandes y el cambio en la proporción de sexos inmediatamente después del pico reproductivo podría ser evidencia de que la población presenta una migración asincrónica, en la cual los machos remontarían río arriba un par de meses antes que las hembras.
Un comportamiento similar se registró en Prochilodus lineatus en el río Uruguay, donde los machos migraron meses antes que las hembras después de reproducirse (Sverlij, et al., 1993).

Por otra parte, mientras que la presencia de hembras maduras y en reposo (EMS III y IV) se registró durante todo el estudio en la ciénaga de Marriaga, no hubo presencia de machos adultos en estos estadios entre abril y julio; este comportamiento se ha observado en poblaciones de especies migratorias en las que los individuos residentes no requieren el desplazamiento migratorio para desencadenar la maduración de sus gónadas (Jonsson \& Jonsson, 1993).

La migración parcial no es rara en las especies de Prochilodus: en Brasil P. argenteus se considera una especie migratoria parcial, pues una parte de la población es residente y permanece en el mismo territorio durante las épocas de desove y las demás (Godinho \& Kynard, 2006). En la zona aluvial de la Guayana venezolana se demostró que las hembras de $P$. mariae residentes y las migratorias maduraban durante la misma temporada (Saldaña \& Venables, 1983). La decisión de migrar o permanecer en las ciénagas podría depender de la oferta alimentaria del lugar, el riesgo de depredación y el gasto energético que pueda demandar el proceso de migración (Jonsson \& Jonsson, 1993; Naslund, et al., 1993; Barbarino-Duque, et al., 1998; Chapman, et al., 2011).

La mortalidad de $P$. magdalenae en la ciénaga de Marriaga $(M=0,97)$ fue inferior a la estimada para otras cuencas de Colombia, por ejemplo, en la cuenca del Magdalena se calculó en $\mathrm{M}=2,29$ año $^{-1}$ (Barreto \& Borda, 2008), caso evidente de una población gravemente sobreexplotada (Galvis \& Mojica, 2007), y en la cuenca del Sinú se calculó en $\mathrm{M}=2,69$ año $^{-1}$ (Valderrama \& Solano, 2004).

El coeficiente de crecimiento $(\mathrm{K})$ para la especie en la ciénaga de Marriaga indica un crecimiento rápido y superior a los registrados en las cuencas del Magdalena ( 0,33 año ${ }^{1}-0,49$ año $\left.^{-1}\right)($ Valderrama \& Petrere, 1994; Barreto 
\& Borda, 2008), del Sinú $\left(0,32\right.$ año $\left.{ }^{-1}\right)$ (Valderrama \& Solano, 2004) y del Atrato $\left(0,24\right.$ año $\left.^{-1}\right)$ (Barreto, et al., 2009). Asimismo, los parámetros de la ecuación de Von Bertalanffy evidenciaron que la población de la ciénaga de Marriaga presentaba una longitud asintótica $\left(\mathrm{L}_{\mathrm{oo}}\right)$ mayor a la registrada para otras cuencas (Magdalena: $\mathrm{L}_{\mathrm{oo}}=50,0$; Sinú: $\mathrm{L}_{\mathrm{oo}}=50,2$ y Atrato: $\mathrm{L}_{\mathrm{oo}}=39,6$ ) (Valderrama \& Solano, 2004; Barreto \& Borda, 2008; Barreto, et al., 2009). Según Sparre \& Venema (1997), la mayoría de las especies de ciclo de vida corto presentan un valor alto de $\mathrm{K}$, y es posible que las condiciones ambientales de la ciénaga de Marriaga le permitan a la población crecer más y con mayor rapidez que en otras cuencas transandinas y zonas del río Atrato, y por ello soportarían aún la extracción pesquera.

\section{Conclusiones}

Teniendo en cuenta la TMM, la LE y la mortalidad, en la ciénaga de Marriaga $P$. magdalenae actualmente se encuentra en mejor estado que las poblaciones de las otras cuencas.

La presencia de hembras adultas durante el año de estudio (a diferencia de los machos), sugiere la existencia de una población residente que permanece en la ciénaga a pesar de los cambios en el régimen hidrológico.

\section{Información suplementaria}

Figura 1S. Distribución de tallas de $P$. magdalenae capturados en la ciénaga de Marriaga, cuenca baja del río Atrato, Colombia. Vea la figura 1S en: https://www.raccefyn.co/index.php/raccefyn/ article/downloadSuppFile/729/2966

Figura 2S. Relación longitud-peso para hembras y machos de $P$. magdalenae capturados en la ciénaga de Marriaga, cuenca baja del río Atrato, Colombia. Vea la figura $2 \mathrm{~S}$ en: https://www.raccefyn.co/ index.php/raccefyn/article/downloadSuppFile/729/2967

Figura 3S. Índice Gonadosomático (IGS) para las hembras de $P$. magdalenae de la ciénaga de Marriaga y pluviosidad, cuenca baja del río Atrato, Colombia. Vea la figura 3S en: https:/www.raccefyn. co/index.php/raccefyn/article/downloadSuppFile/729/2968

Figura 4S. Estimación de la mortalidad por el método de curva linealizada para la los individuos de $P$. magdalenae capturados en la ciénaga de Marriaga, cuenca baja del río Atrato, Colombia. Vea la figura 4S en: https://www.raccefyn.co/index.php/raccefyn/ article/downloadSuppFile/729/2969

Figura 5S. Curva de crecimiento $(\mathrm{Rn}=0,308)$ para los individuos de $P$. magdalenae capturados en la ciénaga de Marriaga, cuenca baja del río Atrato, Colombia.Vea la figura $5 \mathrm{~S}$ en: https://www.raccefyn. co/index.php/raccefyn/article/downloadSuppFile/729/2970

\section{Agradecimientos}

La investigación se llevó a cabo con el apoyo económico y logístico de WWF-Colombia y del Grupo de Investigación en Zoología de la Universidad del Tolima, lo cual fue fundamental para la culminación del estudio.

\section{Contribución de los autores}

Margarita Maria Roa-Cubillos: revisión de muestras, análisis de la información, escritura del manuscrito; Francisco Antonio Villa-Navarro: análisis de la información y escritura del manuscrito.

\section{Conflicto de intereses}

Ninguno.

\section{Referencias}

Allan, J. D., Abell, R., Hogan, Z. E. B., Revenga, C., Taylor, B. W., Welcomme, R. L., \& Winemiller, K. (2005). Overfishing of inland waters. AIBS Bulletin. 55 (12): 1041-1051.

Alonso, J. C., Escobar, F. D., Polo, C. J., Puentes, V. (2014). Aguas Continentales. En: Estado de los principales recursos pesqueros de Colombia - 2014. Serie Recursos Pesqueros de Colombia - AUNAP. Puentes, V., Escobar, F. D., Polo, C. J., y Alonso, J. C. (Eds.). Oficina de Generación del Conocimiento y la Información, Autoridad Nacional de Acuicultura y Pesca - AUNAP. p. 164-199.

Barbarino-Duque, A., Taphorn, D. C., Winemiller, K. O. (1998). Ecology of the Coporo, Prochilodus mariae (Characiformes, Prochilodontidae), and status of annual migrations in western Venezuela. Environmental biology of fishes. 53: 33-46.

Barreto, C. G. \& Borda, C. A. (2008). Evaluación de los recursos pesqueros colombianos. Subgerencia de pesca y acuicultura. Dirección técnica de regulación. Bogotá, D.C. 126 p.

Barreto, C., Borda, C., Otto, J., Sánchez, C., Sanabria, A., Muñoz, S. (2009). Propuesta de cuotas de aprovechamiento de los recursos pesqueros colombianos y ornamentales para la vigencia 2010. Instituto Colombiano Agropecuario ICA. 113 p.

Cala, P. \& Román-Valencia, C. (1999). Cambios cíclicos histomorfológicos de las gónadas del Prochilodus magdalenae (Pisces: Curimatidae), río Atrato, Colombia. Dahlia Rev. Asoc. Colomb. Ictiol. 3: 3-16.

Chapman, B. B., Skov, C., Hulthen, K., Brodersen, J., Nilsson, P. A., Hansson, L.-A., Bronmark, C. (2011). Partial migration in fishes: Definitions, methodologies and taxonomic distribution. Journal of Fish Biology. 81: 479-499.

Codechocó. (2013). Acuerdo consejo directivo número 002. Subdirección de desarrollo sostenible. República de Colombia. $20 \mathrm{p}$.

Di Rienzo, J. A., Casanoves, F., Balzarini, M. G., González, L., Tablada, M., Robledo, C. W. InfoStat versión (2013). Grupo InfoStat, FCA, Universidad Nacional de Córdoba, Argentina. http://www.infostat.com.ar

Froese, R. (2006). Cube law, condition factor and weight-length relationships: History, meta-analysis and recommendations J. Appl. Ichthyol. 22: 241-253.

Galvis, G. \& Mojica, J. I. (2007). The Magdalena river freshwater fishes and fisheries. Aquatic ecosystem health \& management. 10 (2): 127-139.

Gayanilo, F. C, \& Pauly, D. 1997. FAO - ICLARM stock assessment tools. Reference manual. Computarized Information Series. FAO. Rome.

González, E., Varona, M., Cala, P. (1996). Datos bioecológicos del oscar, Astronotus Ocellatus (Pisces: Cichlidae), en los alrededores de Leticia, Amazonía. Dahlia. Rev. Asoc. Colomb. Ictiol. Santa fe de Bogotá. 1: 51-62. 
Godinho A. L. \& Kynard, B. (2006) Migration and spawning of radio-tagged zulega Prochilodus argenteus in a dammed Brazilian river. Transactions of the American Fisheries Society. 135: 811-824.

Gutiérrez F. de P., Rivas-Lara, T. S., Rincón López, C. (2011). Diagnóstico de la pesquería en la cuenca del Atrato. Capítulo 3. 103-118 p. En: Lasso, C. A., F. de Paula Gutiérrez, M. A. Morales-Betancourt, E. Agudelo, H. Ramírez-Gil y R. E. Ajiaco-Martínez (Eds). (2011). II. Pesquerías continentales de Colombia: cuencas del Magdalena-Cauca, Sinú, Canalete, Atrato, Orinoco, Amazonas y vertiente del Pacífico. Serie Editorial Recursos Hidrobiológicos y Pesqueros Continentales de Colombia. Instituto de Investigación de los Recursos Biológicos Alexander von Humboldt. Bogotá, D. C., 304 p.

INVEMAR. (2002). Informe anual sobre el estado de los ambientes marinos y costeros en Colombia, Programa Sinam - Unidad coordinadora de información. INVEMAR. $148 \mathrm{p}$.

Jaramillo-Villa, U. \& Jiménez-Segura, L. F. (2008). Algunos aspectos biológicos de la población de Prochilodus magdalenae en las ciénagas de Tumaradó (río Atrato), Colombia. Actual. Biol. 30 (88): 55-66.

Jonsson, B. \& Jonsson, N. (1993). Partial migration: Niche shift versus sexual maturation in fishes. Reviews in Fish Biology and Fisheries. 3: 348-365.

Maldonado-Ocampo, J. A., Usma, J. S., Villa-Navarro, F. A., Ortega-Lara, A., Prada-Pedreros, S., Jiménez, L.F., Jaramillo-Villa, U., Arango, A., Rivas, T., Sánchez, G. C. (2012). Peces dulceacuícolas del Chocó Biogeográfico de Colombia. WWF Colombia, Instituto de Investigación de Recursos Biológicos Alexander von Humboldt (IAvH), Universidad del Tolima, Autoridad Nacional de Acuicultura y Pesca (AUNAP), Pontificia Universidad Javeriana. Bogotá D.C., Colombia. 400 p.

Mojica J. I., Valderrama, M., Barreto, C., Álvarez-León, R. (2012). Prochilodus magdalenae. En: Mojica, J. I., J. S. Usma, R. Álvarez-León \& C. A. Lasso (Eds). (2012). Libro rojo de peces dulceacuícolas de Colombia 2012. Instituto de Investigación de Recursos Biológicos Alexander von Humboldt, Instituto de Ciencias Naturales de la Universidad Nacional de Colombia, WWF Colombia y Universidad de Manizales. Bogotá, D. C., Colombia, 319 p.

Mora, J.M. (1990). Impacto ambiental de la pesca sobre el complejo de ciénagas de Tumaradó Parque Nacional Natural Katios, Chocó-Colombia. Trabajo de grado. Universidad Jorge Tadeo Lozano. Bogotá D.C.

Naslund, I., Milbrink, G., Eriksson, L. O., Holmgren, S. (1993). Importance of habitat productivity differences, competition and predation for the migratory behaviour of Arctic charr. Oikos. 66: 538-546.

Parque Nacional Natural de Los Katíos. (2006). Plan de manejo Parque Nacional Natural Los Katios 2005-2009. Unidad Administrativa Especial del Sistema de Parques Nacionales Naturales de Colombia. Territorial Norocccidental. TurboAntioquia. 275 p.
Rivas, T., Conte, B., Puentes, E., Cuesta, E., Mena, M., Ramírez, A., Blandón, M., Casas, Y., Carrascal, O., Rivas, W. (2002). Estado actual de la pesca en la cuenca media del rio Atrato, Chocó-Colombia. Revista Universidad Tecnológica del Choco. 15: 25-32.

Saldaña J. \& Venables B. (1983). Energy compartmentalization in migratory fish, Prochilodus mariae (Prochilodontidae) of the Orinoco River. Copeia. 3: 617-623.

Sverlij, S. B., Espinach A., Orti, G. (1993). Sinopsis de los datos biológicos y pesqueros del sábalo Prochilodus lineatus (Valenciennes, 1847). FAO Sinopsis sobre la Pesca, No.154. Roma, FAO. 64 p.

Sparre P. \& Venema, S. C. (1997). Introducción a la evaluación de los recursos pesqueros tropicales. Parte 1. FAO Documento técnico de pesca. $\mathrm{N}^{\circ} 306.1$, Rev.2. Roma.

Valderrama, M., Mogollón, M., Solano, D., Álvarez, L. (2002). Monitoreo y estadística pesquera en la cuenca del rio Sinú con participación comunitaria. Informe Final, periodo marzo 2001 febrero 2002, presentado a Urra SA E.5.P. Montería. Colombia.

Valderrama, M. \& Petrere, M. Jr. (1994). Crecimiento del bocachico Prochilodus magdalenae (Steindachner, 1878; Prochilodontidae) y su relación con el régimen hidrológico en la parte baja de la cuenca del río Magdalena (Colombia). Boletín Científico INPA. 2: 136-152.

Valderrama, M. \& Solano, D. (2004). Estado de la población de bocachico, Prochilodus magdalenae (Pisces: Characiformes), y su manejo en la cuenca del río Sinú, Colombia. Dahlia, Rev Asociación Colombiana de Ictiología. 7: 3-12.

Valderrama, M., Jiménez-Segura, L.F., López-Casa, S., Rivas, T. S., Rincón, C. E., Nieto-Torres, S., González-Cañón, G., Galvis-Galindo, I., Hernández-Barrero, S., Salas F.P. (2011). Prochilodus magdalenae (Prochilodontidae). Capítulo 7,8. 305-311p. En: Lasso, C. A., E. Agudelo Córdoba, L. F. Jiménez-Segura, H. Ramírez-Gil, M. Morales-Betancourt, R. E. Ajiaco-Martínez, F. de P. Gutiérrez, J. S. Usma, S. E. Muñoz Torres y A. I. Sanabria Ochoa (Eds.). I. Catálogo de los recursos pesqueros continentales de Colombia. Serie Editorial Recursos Hidrobiológicos y Pesqueros Continentales de Colombia. Instituto de Investigación de los Recursos Biológicos Alexander von Humboldt (IAvH). Bogotá, D.C., 715 p.

Vazzoler, A. E. A. de M. (1996). Biologia da reprodução de peixes teleosteos: teoria e pratica. EDUEM, Maringa, Sao Pablo, $169 \mathrm{p}$.

Welcomme R. L. (1999). A review of a model for qualitative evaluation of exploitation levels in multi-species fisheries. Fisheries Management and Ecology. 6: 1-19.

Wootton, R. (1998). Ecology of teleost fishes. $2^{\circ}$ ed. Kluwer Academic Publishers. Boston, 386 p. 